\title{
Práticas de Ensino-Aprendizagem e de Pesquisa com Abordagem Qualitativa Interdisciplinar para Conscientização e Conhecimento do Fenômeno Bullying
}

\author{
Maria Aparecida Miranda de Paula Machado*, Gessyka Gomes Marcandal*, Ariadnes \\ Nóbrega de Oliveira*
}

Departamento de Fonoaudiologia, Faculdade de Odontologia de Bauru, Universidade de São Paulo

*Autoras para correspondência: cidamachado@usp.br; gessyka@usp.br; dine_usp@yahoo.com.br

\begin{abstract}
RESUMO
Práticas de ensino-aprendizagem e de pesquisa qualitativa interdisciplinar foram empregadas nas atividades de formação acadêmica em cenários reais para estudar o fenômeno bullying. Levar todos os envolvidos a vivenciarem essas práticas em atividades de integração entre universidade-escolas públicas de nível básico e conscientizar sobre a existência e formas de enfrentamento do bullying foram os objetivos previstos. Este artigo é um relato de experiência de caráter qualitativo e interdisciplinar sobre trinta oficinas que ocorreram entre 2011 e 2013, abrangendo 59 professores, cinco gestores, doze estudantes de ensino fundamental - ciclo II e treze funcionários da equipe de apoio escolar, inscritos por demanda espontânea; por outro lado, um docente responsável pelas pesquisas, duas fonoaudiólogas da equipe universitária, duas estudantes de ensino médio de Pré-Iniciação Científica, duas graduandas em Fonoaudiologia de Iniciação Científica e quatro acadêmicos colaboradores. Durante as oficinas foram produzidos grupos focais, debates, instrumentos de observação e de entrevista. Os graduandos de Fonoaudiologia participantes, em cada ano, foram incluídos em reuniões prévias e de acompanhamento das oficinas e na aplicação das práticas dialéticas de ensino-aprendizagem. Foi observado que os estudantes do nível básico e superior participaram e acompanharam os debates efetiva e ativamente; um Roteiro de Discussão sobre o tema foi elaborado e distribuído para as escolas municipais; com os dados de observação e entrevistas, programas de redução do bullying nas escolas participantes foram aperfeiçoados ou criados. Houve sensibilização, conscientização e desenvolvimento de conhecimento, sendo aprimoradas as competências e habilidades de cada grupo participante.
\end{abstract}

Palavras-Chave: Pesquisa Interdisciplinar; Formação Profissional em Saúde; Formação Profissional; Educação Permanente; Bullying.

\begin{abstract}
Teaching-learning practices and research with qualitative interdisciplinary approach were employed in academic training activities in real scenarios to study the phenomenon of bullying. The aims were to lead all those involved to experience these practices in activities of integration between university-public elementary schools and awareness about the existence and forms of facing to bullying. This article, report of experience of qualitative and interdisciplinary characteristics about 30 workshops occured between 2011 and 2013, embraced 59 teachers, five managers, 12 elementary school students - cycle II and 13 staff of the school support team, enrolled by spontaneous demand, still, a professor in charge of research, two speech therapists of the university team, two high school students of PreScientific Initiation, two undergraduate students in Speech, Language and Hearing Science of Scientific Initiation and four academic collaborators. During the workshops, focal groups, debates, and the elaboration of observation and interview tools were produced. Students of Speech, Language and Hearing Science, each year, were included in previous meetings and follow-up workshops and the application of dialectical teaching-learning practices. It was observed that the students of the basic and university level participated and followed the debates effectively and actively; a Discussion Script on the theme was prepared and distributed to municipal schools; with observation data and interviews bullying reduction programs of participating schools were improved or created. There was awareness and knowledge development, and the skills of each participating group were improved.
\end{abstract}

Keywords: Interdisciplinary Research; Health Human Resource Training; Professional Training; Education Continuing; Bullying. 


\section{Introdução}

Adotar noções de interdisciplinaridade nas práticas de ensino-aprendizagem e de pesquisa qualitativa significa responder a desafios e conflitos de proporções complexas "[d]aquilo que é tecido conjuntamente, [d]aquilo que se deve enlaçar" (MORIN, 2007, p. 22), caracterizando a operacionalização de procedimentos, informações e recursos de mais de uma disciplina (JAPIASSU, 2006, p. 101). Portanto, consiste na "troca de conceitos, teorias e métodos entre as diferentes disciplinas" (TEIXEIRA, 2007, p. 72).

A partir dessas premissas, foi iniciado o planejamento de oficinas de estudo e pesquisa, que contemplasse a construção conjunta do conhecimento a respeito do complexo envolvendo o bullying, tema solicitado pela Secretaria Municipal de Educação. O assunto é polêmico e muitas vezes não admitido pelo sistema educacional. Assim, foram previstas a utilização da técnica de grupo focal e a elaboração de instrumentos de observação e de entrevista para pesquisa. Os debates ocorreram durante o grupo focal e nas discussões dos dados obtidos.

Na Europa não há uma única definição para o termo bullying, visto que dependerá do tipo de ato violento que é produzido, contudo o consenso é que se trata de violência na escola (SMITH, 2003, p. 138). Dessa forma, é explicado como "fenômeno pelo qual uma criança ou um adolescente é sistematicamente exposta(o) a um conjunto de atos agressivos (diretos ou indiretos), que ocorrem sem motivação aparente, mas de forma intencional, protagonizados por um(a) ou mais agressor(es)" (LISBOA et al., 2009, p. 60). Esses eventos incidem dentro, no entorno ou entre pessoas de uma mesma comunidade escolar e são caracterizados por desequilíbrio de poder e ausência de reciprocidade, situação em que a vítima possui pouco ou quase nenhum recurso para evitar e/ou defender-se da agressão (NETO, 2005, p. 165). A interação grupal é reforçada ou enfraquecida por uma complexa rede de articulação entre estímulos aos quais estão expostos os jovens, as famílias e as escolas, permeados pelo contexto sociocultural (ALMEIDA et al., 2007, p. 108) e, se não identificada e evitada, pode produzir sérios danos psicológicos e pedagógicos para os envolvidos, tanto vítimas como agressores (TRAUTMANN, 2008, p. 15).

É necessário, portanto, que os professores e demais profissionais vinculados à instituição escolar estejam atentos para discernir uma situação normal de brincadeira e interromper um episódio de bullying. Para esse fim, o processo de intervenção pedagógica requer atuação interdisciplinar, em função do "movimento da realidade e construção/ produção do conhecimento" (BICUDO, 2008, p. 146) que permeiam os aspectos presentes nas condições de vida e dos direitos sociais dos aprendizes. Essa intervenção recupera, para ambas as partes - educador e estudante - conhecimentos e comportamentos complexos e diversificados de dimensão intersubjetiva da interação social, os quais transcendem o campo da educação (Idem, p.147). Assim, a formação permanente interdisciplinar desses profissionais traz outros saberes que os sustentam nesse processo de interação cotidiana no reconhecimento das necessidades da comunidade, do qual fazem parte a intervenção e a prevenção desse fenômeno (SANTOS, 2007, p. 21).

A temática preventiva a respeito da prática do bullying tem sido assunto bastante discutido, no entanto a literatura traz poucos estudos sobre promoção de ambientes saudáveis e pacíficos que possibilite a efetiva participação dos estudantes em atividades reflexivas, geradoras de argumentação crítica sobre o assunto e o impacto que essa tecnologia metodológica possa ocasionar no ambiente escolar e familiar.

Considerando o recorte da promoção da saúde, os objetivos do estudo foram de conscientizar a comunidade escolar da Rede de Educação Pública de um município do Sudeste do Brasil, quanto à existência e a formas de enfrentamento do bullying e oportunizar a vivência de práticas em pesquisas qualitativas aos estudantes da graduação do curso de Fonoaudiologia inseridos em projetos de Iniciação Científica.

\section{Método}

Os projetos foram submetidos ao Comitê de Ética em Pesquisa em Seres Humanos, gerando 
dois distintos processos: n. 26/2011 e n. 145/2011, e as pesquisas envolveram professores, gestores da educação, estudantes do Ensino Fundamental - ciclo II, equipe de apoio escolar e estudantes de ensino médio e de graduação.

Classificado como Relato de Experiência, fundamentado em pesquisa de abordagem qualitativa de intervenção e interdisciplinar (MINAYO, 2010, p. 163), este estudo fez uso de metodologias dialéticas com categorias conceituais comparativas na literatura, na análise dos dados.

As oficinas ocorreram em um total de trinta, com quatro horas cada uma, em 2011, com 41 professores; em 2012, com dezoito professores, cinco gestores da Secretaria de Educação Municipal e doze estudantes do Ensino Fundamental - ciclo II, e em 2013 com oito funcionárias da limpeza e cinco merendeiras, sendo a inscrição por demanda espontânea.

Quando se trata da graduação, em 2011 houve a participação de um estudante de iniciação científica (IC) e auxílio para inclusão no acervo de dados de mais dois acadêmicos colaboradores do curso de Fonoaudiologia (ACF). Em 2012, além de uma IC, um ACF contribuiu com os gráficos de análise dos dados, e dois estudantes de ensino médio, provenientes de um edital de Pré-Iniciação Científica (PIC), também participaram das reuniões e da pesquisa. Em 2013, dois ACF colaboraram com a análise dos dados. Para cada ano os estudantes foram incluídos em reuniões prévias, de acompanhamento das oficinas e da aplicação das práticas dialéticas de ensino.

Durante as oficinas foi utilizada a técnica de grupo focal exploratório, caracterizada pela interação e sondagem dos pontos de vista, como parte integrante do método, "a partir de reflexões sobre um determinado fenômeno social [...], gerando suas próprias perguntas e buscando respostas pertinentes à questão sob investigação" (BACKES et al., 2011, pp. 438-439). É um recurso para "compreender o processo de construção das percepções, atitudes e representações sociais de grupos humanos" (GONDIM, 2003, p. 151).

Os grupos focais tiveram a duração média de noventa minutos e duas questões disparadoras de debate - "Fale sobre o que é bullying para você" e "Quais desafios você encontra para trabalhar com os estudantes a respeito deste tema". As intervenções didáticas sobre o assunto foram fundamentadas em literatura científica e artística, recomendadas como leitura complementar.

$\mathrm{Na}$ sequência, e durante as oficinas, houve a construção dos instrumentos de pesquisa, denominados de roteiros de observação e de entrevista. Os roteiros têm a finalidade de abranger as multifaces do objeto de investigação, facilitando sua operacionalização (MINAYO, 2010, p. 189).

Assim, os Roteiros de Observação tiveram a finalidade de "limitar e definir com precisão os objetivos que se deseja alcançar" (QUEIROZ et al., 2007, p. 277), sob o ponto de vista do observador. Foram preenchidos por professores, gestores e equipe de apoio das Escolas Municipais de Ensino Infantil e Infantil Integral (EMEI e EMEII), que compreendem crianças entre zero e cinco anos e onze meses e de Escolas Municipais de Ensino Fundamental, $1^{\circ}$ e $2^{\circ}$ níveis (EMEF), com crianças na faixa etária de seis anos a onze anos e onze meses, e de adolescentes entre doze e dezessete anos.

No caso da Entrevista, "definida como um processo de interação social [...], em que o entrevistador tem por objetivo a obtenção de informações do entrevistado" (HAGUETE, 2007, p. 86), o roteiro semiestruturado orientou o processo de comunicação e destacou os indicadores essenciais para o estudo do objeto (MINAYO, 2010, p. 191). Essa modalidade foi aplicada em turmas de adultos em um Centro Educacional de Jovens e Adultos (CEJA) e de estudantes de cada sala do segundo ciclo, eleitos de forma aleatória. Evidenciou questões referentes aos dados pessoais (sexo, etnia, origem, escolaridade, profissão, composição e dinâmica familiar, relação comunitária, lazer, acesso aos meios de comunicação) e dados de ambiente escolar.

Convém esclarecer que esses instrumentos foram aplicados nas escolas que eram representadas por professores nas oficinas, com aceite e assinatura do termo de Consentimento Livre e Esclarecido por alunos adultos ou por 
representantes legais das crianças e adolescentes convidados.

Os roteiros foram elaborados conjuntamente entre os participantes de cada oficina, no seu ano de ocorrência, e o docente responsável pela pesquisa, sua equipe e discentes com IC e PIC. Esses estudantes participaram ativamente de todo o processo, na busca e no inventário das referências, bem como na aprendizagem para discussão do tema durante os debates, na elaboração desses instrumentos de pesquisa, e alguns, na elaboração de um Roteiro para Debate nas escolas municipais e na análise dos dados.

A coleta de dados foi acompanhada de maneira prévia, durante e posteriormente, por meio de discussão em seminários gerais previstos nas oficinas.

\section{Resultados e Discussão}

No total foram entrevistados doze alunos de CEJA, 65 alunos de EMEF, e obtidos 75 roteiros de observação. $O$ roteiro de observação realizado pelos professores, levando-se em conta as mais variadas situações e momentos diários dos alunos nos diversos ambientes, e as entrevistas foram fundamentais para obter informações a respeito do fenômeno, possibilitando sua detecção em todos os níveis de ensino. Consequentemente, as abordagens foram se somando para delinear a compreensão do fenômeno bullying, e as oficinas puderam propiciar profunda reflexão sobre o assunto e sobre o impacto que produz no ambiente escolar.

Em qualquer um dos níveis de ensino, a maioria dos professores mostrou os meios, conhecimentos e disposição para intervir, quanto aos comportamentos e atitudes, de forma didática e afetiva. Esses dados são corroborados por estudiosos quando referem a importância do trabalho com a diversidade, consideram a reflexão como um caminho positivo para a redução do bullying e a promoção de condições adequadas para que o estudante desenvolva seus potenciais e habilidades de forma saudável (CARVALHO \& SILVA, 2011, p. 97).

A maioria dos estudantes referiu a existência do bullying na escola onde estuda, mas a minoria relatou reagir de forma violenta, fato que confirma a observação de uma parcela significativa de autores, segundo os quais grande porcentagem das crianças vítimas de bullying não reage, ou entra em sofrimento sem comentar com qualquer adulto (BRINO, 2015; OLIVEIRA-MENEGOTTO et al., 2013; NETO, 2005).

A maioria dos estudantes entrevistados, vítimas e/ou agressores, referiu não apresentar dificuldades de relacionamento familiar e com amigos. Embora a forma como se dava o relacionamento dessas crianças e adolescentes não tenha sido comprovada pela inserção dos familiares nas oficinas, estes puderam ser observados nas interações com outros colegas, e seria possível justificar os agressores, em parte, pelo que foi considerado como "autores de bullying que admitiram não terem sido advertidos" (NETO, 2005, p. 166).

Em 2012, cada escola elencou os programas que incluíam o estudante em políticas da gentileza, reflexão e cultura da paz para serem discutidos e implementados. Todas as escolas participantes apresentaram ao menos três programas, construíram outros no decorrer do ano e aprovaram a liderança de estudantes nas ações, sustentados por professores que se disponibilizaram, como forma de conscientização e empoderamento. Criaram uma agenda para discussão local e foi construído e publicado, conjuntamente ao grupo de estudantes (IG, ACF e PIC), um Roteiro para Debate, distribuído para toda a Rede Municipal.

Vários programas de redução do bullying foram divulgados nas redes sociais (blogs, fóruns, portais, entre outros) nos últimos anos e implantadas algumas leis municipais, no país. No entanto, a prática de inclusão dos estudantes nas discussões e reflexões, na liderança dos projetos e programas em que se adotam autonomia própria, defesa de ideias e de argumentos, e responsabilidades com o meio, foi surgindo com potência durante as oficinas. A manifestação dos estudantes de graduação e de ensino fundamental e médio, nesse ínterim, foi essencial para polemizar os processos de inclusão e liderança e serviu como exercício para os professores lidarem com essas questões na implantação dos programas de redução do bullying. 
Em 2013 a equipe de apoio observou os alunos a partir dos seus pontos estratégicos de trabalho, o que oportunizou argumentação deflagrada por meio dos mitos e verdades a respeito do bullying, equiparou conhecimentos, desenvolveu a confiança e destacou os comportamentos agressivos vistos como naturais, até então, e ignorados ou pouco valorizados por todos os envolvidos na comunidade escolar. Desde as oficinas, alguns profissionais da equipe de apoio passaram a fazer parte dos programas que ocorriam nas escolas onde trabalhavam. Não foram encontrados artigos referentes à prática com a equipe de apoio, entretanto é possível justificar a adoção das ações pedagógicas educativas articuladas dentro de um processo de gestão democrática, tomando a escola como "um agente chave na transformação para uma sociedade mais justa e igualitária" (CARVALHO \& SILVA, 2011, p. 97).

As limitações com as quais as pesquisas realizadas se depararam estão relacionadas com: a falta de acesso a dados prévios referentes ao processo de bullying em cada escola para termos comparativos posteriores; a ausência de inclusão das famílias dos estudantes nos debates e reflexões; e a dificuldade de inclusão de todos os graduandos no processo.

Entretanto, destaca-se o processo de provocação de consciência, sobre o objeto, para estudantes de todos os níveis de ensino. Também sobressaem os cuidados necessários que os professores tiveram, desde o convite para a entrevista até à liderança dos programas na tentativa de evitar represálias ou hostilização.

Essas pesquisas de intervenção buscaram contribuir para ampliar a discussão da temática em espaços de integração universidade-serviçoscomunidade, com base nos princípios que regem o sistema único de saúde, destacando o papel social das instituições de ensino.

\section{Considerações Finais}

Os resultados relativos à sensibilização, conscientização e constituição dialética do conhecimento puderam ser observados processualmente, no decorrer das atividades. As competências comunicativas de cada grupo foram aprimoradas, graças à abordagem interativa adotada. Nos procedimentos de construção e emprego dos instrumentos de observação e entrevista e na implantação das ações e programas, destacamse: a sobreposição do conhecimento do processo pedagógico com o qual os professores e gestores atuaram; as atitudes respeitosas, valores e comportamentos que as crianças e adolescentes apresentaram; a observação empírica e afetiva que transversalizou o discurso da equipe de apoio; e o exercício de cidadania, política e interação comunicativa potencializada que os fonoaudiólogos proporcionaram.

Quanto aos estudantes da graduação, houve valorização das abordagens qualitativas, e a experiência foi replicada em outros contextos de extensão universitária. Referiram que a vivência trouxe escuta e olhar mais humanizado aos projetos de pesquisa e de interação clínica. Além disso, a construção do conhecimento, conteúdo concernente à disciplina de Saúde Coletiva, foi prazerosa e significativa para as dimensões de formação profissional, ética e pessoal.

Sugere-se a realização de pesquisas de intervenção incluindo famílias e comunidades.

\section{Referências Bibliográficas}

ALMEIDA, Ana; LISBOA, C. \& Caurcel, M. "¿Porqué Ocurren los Malos Tratos entre Iguales? Explicaciones Causales de Adolescentes Portugueses y Brasileños". Revista Interamericana de Psicología, Porto Alegre, vol. 41, n. 2, 2007, pp. 107-118.

BACKES, Dirce Stein; COLOMÉ, J. S.; ERDMANN, R. H. \& LUNARDI, V. L. "Grupo Focal como Técnica de Coleta e Análise de Dados em Pesquisas Qualitativas". O Mundo da Saúde, São Paulo, vol. 35, n. 4, 2011, pp. 438-442.

BICUDO, Maria Aparecida Viggiani. "A Pesquisa Interdisciplinar: uma Possibilidade de Construção do Trabalho Científico/Acadêmico". Educaşão Matemática Pesquisa, São Paulo, vol. 10, n. 1, 2008, pp. 137-150.

BRINO, Rachel de Faria; LIMA, M. H. G. G. "Compreendendo Estudantes Vítimas de Bullying: Para quem eles Revelam?". Psicologia da Educação, São Paulo, n. 40, 2015, pp. 27-39.

CARVALHO, Alba Ataciane de Lima Carvalho \& 
SILVA, M. L. "O Bullying e a Gestão Democrática de Escolas Públicas: Algumas Reflexões". Olhares Plurais, Maceió, vol. 1, n. 4, 2011, pp. 81-98.

GONDIM, Sônia Maria Guedes. "Grupos Focais como Técnica de Investigação Qualitativa: Desafios Metodológicos". Paidéia, Ribeirão Preto, vol. 12, n. 24, 2003, pp. 149-161.

HAGUETE, Teresa Maria Frota. Metodologias Qualitativas na Sociologia. Petrópolis: Vozes, 2007.

JAPIASSU, Hilton. O Sonho Transdisciplinar $e$ as Razões da Filosofia. Rio de Janeiro: Imago, 2006.

LISBOA Carolina; BRAGA, L. L. \& EBERT, G. "O Fenômeno Bullying ou Vitimização entre Pares na Atualidade: Definições, Formas de Manifestação e Possibilidades de Intervenção". Contextos Clínicos, São Leopoldo, vol. 2, n. 1, jan./jun. 2009, pp. 59-71.

MINAYO, Maria Cecília de Souza. O Desafio do Conhecimento: Pesquisa Qualitativa em Saúde. São Paulo: Hucitec, 2010.

MORIN, Edgar. "Desafios da Transdisciplinaridade e da Complexidade". In: AUDY, Jorge Luis Nicolas et al. (orgs.). Inovação e Interdisciplinaridade na Universidade. Porto Alegre: EDIPUCRS, 2007, pp. 22-28.

NETO, Aramis Lopes. "Bullying: Comportamento Agressivo entre Estudantes". Jornal de Pediatria, Porto
Alegre, vol. 81, n. 5, supl., 2005, pp. S165-172.

OLIVEIRA-MENEGOTTO, Lisiane Machado; PASINI, A. I.; LEVANDOWSKI, G. "O Bullying Escolar no Brasil: uma Revisão de Artigos Científicos". Revista Psicologia: Teoria e Prática, São Paulo, vol. 15, n. 2, maio/ago. 2013, pp. 203-215.

QUEIROZ, Danielle Teixeira; VALL, J.; SOUZA, Â. M. A. \& VIEIRA, N. F. C. "Observação Participante na Pesquisa Qualitativa: Conceitos e Aplicações na Área da Saúde". Revista Enfermagem Uerj, Rio de Janeiro, vol. 15, n. 2, abr./jun. 2007, pp. 276-283.

SANTOS, Luciana Pavan Ribeiro. O Papel do Professor Diante do Bullying na Sala de Aula. Trabalho de Conclusão de Curso, Faculdade de Ciências - Unesp, Campus de Bauru- Universidade Estadual Paulista, 2007.

SMITH, Peter. Violence in Schools. The Response in Europe. London, UK: Routledge Falmer, 2003.

TEIXEIRA, Evilázio Francisco Borges. "Emergência da Inter e da Transdisciplinaridade na Universidade". In: AUDY, Jorge Luis Nicolas et al. (orgs.). Inovação e Interdisciplinaridade na Universidade. Porto Alegre: EDIPUCRS, 2007, pp. 58-80.

TRAUTMANN, Alberto M. "Maltrato entre Pares o "Bullying". Una Visión Actual". Revista Chilena de Pediatria, 79, 2008, pp. 13-20.

Publicado em 12/06/2018. 